\title{
IMPACTO DA IRRIGAÇÃO SOBRE OS SOLOS DE PERÍMETROS IRRIGADOS NA BACIA DO ACARAÚ, CEARÁ, BRASIL ${ }^{1}$
}

\author{
JOSÉ F. B. LOPES ${ }^{2}$, EUNICE M. DE ANDRADE ${ }^{3}$, LUIZ C. G. CHAVES ${ }^{4}$
}

\begin{abstract}
RESUMO: Para avaliar o risco de salinidade e de sodicidade do solo pelo manejo da irrigação, desenvolveu-se estudo comparativo entre os valores de CEes e RAS de área irrigada com os da mata nativa. A investigação ocorreu nos Distritos Irrigados Baixo Acaraú (DIBAU) e Araras Norte (DIPAN), localizados na Bacia do Acaraú. As amostras de solo foram coletadas bimestralmente, de maio/2003 a set./2005, nas camadas de 0-0,30 m; 0,30-0,60 m; 0,60-0,90 m e 0,90-1,20 m, sendo as análises efetuadas no Laboratório de Solo e Água da Embrapa Agroindústria Tropical. Para identificar se os incrementos dos parâmetros estudados eram estatisticamente significativos, aplicou-se o teste de Wilcoxon. Os incrementos de CEes foram maiores no DIPAN, com média de 250\%, enquanto no DIBAU, a média atingiu 50\%. Em 2004, as precipitações pluviométricas levaram a área irrigada do DIBAU à condição de mata nativa, não ocorrendo o mesmo no DIPAN. Para a RAS, os incrementos médios situaram-se em torno de 200\% (DIBAU) e não houve incremento no DIPAN. Portanto, os solos do DIBAU apresentaram maior predisposição à sodicidade, e os do DIPAN, maior risco de salinização. Apesar dos incrementos, os valores da CEes e RAS apresentaram-se inferiores ao limite de classificação de solos salinos e sódicos, respectivamente. No DIBAU, a chuva teve maior influência no processo de lixiviação devido à predominância da textura arenosa dos solos.
\end{abstract}

PALAVRAS-CHAVE: salinidade, lixiviação, adsorção de sódio.

\section{IRRIGATION IMPACTS IN THE SOILS AT IRRIGATED DISTRICT IN ACARAU WATERSHED, CEARÁ, BRAZIL}

\begin{abstract}
To identify the soil salinity and sodium risk, due to irrigation management, was carried out a comparative study of the Electric Conductivity (EC) and Sodium Adsorption Ration (SAR) values, between an irrigated area and an undisturbed land $(\mathrm{MN})$. The investigation occurred at two Irrigated Districts: Baixo Acaraú (DIBAU) and Araras Norte (DIPAN) sited in Acaraú watershed, Ceará, Brazil. Soils samples were collected, in 0-30 m; 30-60 m; 60-90 m and 90-120 $\mathrm{cm}$ layers, in bimestrial period, from May/2003 to September/2005. Analyses were carried out at Embrapa Soil and Water Laboratory. To identify if the addition of salts in the soils were statistically significant the Wilcoxon paired test was applied. The EC increments were higher in DIPAN with an average of $250 \%$, while in DIBAU the average reached 50\%. The total rainfall registered in 2004 was enough to guide the irrigated soils in DIBAU to a similar condition of undisturbed land area. The same did not occur with the soil in DIPAN. The medium values of SAR increments in DIBAU were around $200 \%$. An increasing of SAR was not registered at DIPAN. Therefore, the soils of DIBAU showed higher tendency to sodium problems, while the soils at DIPAN presented a higher risk of salinity. Despite of the registered increments, EC and SAR values were lower than those of salinity and sodium soils, respectively. Also, results showed that the action of rainfall in the leaching process of salts was higher at DIBAU, due to the soil texture (sand soils).
\end{abstract}

KEYWORDS: salinity, leaching, sodium adsorption.

\footnotetext{
${ }^{1}$ Trabalho apresentado no XXV Encontro de Iniciação Científica da UFC e selecionado como o melhor artigo da área de Agronomia.

${ }^{2}$ Estudante de Agronomia, Bolsista do PET, Departamento de Engenharia Agrícola, Centro de Ciências Agrárias, UFC, Fortaleza CE, Fone: (0XX85) 3366 9762, fredsonufc@yahoo.com.br

${ }^{3}$ Eng $^{-}$Agrônoma, Profa. Ph. D., Departamento de Engenharia Agrícola, CCA/UFC, Fortaleza - CE.

${ }^{4}$ Tecnólogo em Recursos Hídricos/Irrigação, M. Sc. em Irrigação e Drenagem, Departamento de Engenharia Agrícola, CCA/UFC,Fortaleza - CE.

Recebido pelo Conselho Editorial em: 19-1-2007

Aprovado pelo Conselho Editorial em: 26-11-2007
}

Eng. Agríc., Jaboticabal, v.28, n.1, p.34-43, jan /mar. 2008 


\section{INTRODUÇÃO}

Com o acelerado crescimento populacional mundial e a busca por melhor qualidade de vida das populações, surge, a cada dia, a necessidade de maior produção de alimentos. Com isso, expandiram-se as áreas agricultáveis em todo o mundo, impulsionando, então, o uso da irrigação, não só como complemento das necessidades hídricas das regiões úmidas, mas também como opção de tornar produtivas as regiões áridas e semi-áridas do planeta. ONGLEY (2000) afirma que, com a expansão das áreas irrigadas, crescem também os problemas de degradação dos recursos naturais (solo e água), principalmente aqueles relacionados à salinidade e à sodicidade do solo. Esses problemas ocorrem principalmente em regiões áridas e/ou semi-áridas, onde a precipitação anual não garante a lavagem dos sais acumulados. Assim, a lavagem dos sais na zona radicular deve ser conduzida para manter a concentração salina do solo em um nível aceitável (MEIRELES et al., 2003). Já em regiões onde a precipitação é relativamente alta, a estação chuvosa pode assegurar a lavagem dos sais (BEN-HUR et al., 2001; ANDRADE et al., 2004).

Pesquisadores como SMEDEMA \& SHIATI (2002) afirmam que, mesmo em uma visão conservacionista, de três a cinco toneladas de sais são adicionadas por hectare irrigado por ano, nas regiões secas do globo. SOUZA et al. (2000) reforçam que, em áreas irrigadas, o processo de salinização pode acontecer mesmo em solos com boas características, em especial nas situações em que não existe manejo de solo e água adequado. Na realidade, a concentração de sais nos solos irrigados apresenta relação direta com a precipitação total anual, com as características físicas do solo e com as condições de drenagem. Sob condições favoráveis, esses sais poderão voltar ao rio ou ao aqüífero de origem (PEREIRA et al., 1986; BEN-HUR et al., 2001).

Embora a intemperização dos minerais primários seja a fonte principal e direta de todos os sais solúveis encontrados no solo, raramente tem-se verificado acúmulo de quantidades suficientes de sais solúveis por meio desse fenômeno (salinização primária), uma vez que grande parte dos sais liberados são transportados pelas águas subterrâneas e/ou fluviais até os oceanos (PIZARRO, 1978). Para SILVA FILHO et al. (2000), HOLANDA \& AMORIM (1997), LIMA (1997) e D’ALMEIDA et al. (2005), o processo de salinização dos solos pode não estar ligado diretamente à qualidade da água utilizada na irrigação, dependendo também das características físico-químicas do solo em seu estado natural e do manejo aplicado ao mesmo. Em estudos mais recentes, pesquisadores como CAUSAPÉ et al. (2004) e FENG et al. (2005) vêm identificando elevada adição de sais ao solo pelos fertilizantes químicos.

No Nordeste brasileiro, em torno de $30 \%$ das áreas irrigadas dos projetos públicos estão comprometidas com problemas de salinização (BERNARDO, 1997). D’ALMEIDA (2002) argumenta que, mesmo a irrigação sendo uma prática que data de milênios, a importância de informações sobre o manejo adequado, visando à preservação do meio ambiente, só começou a ser preocupante a partir do século passado, quando se observou acelerado crescimento populacional mundial e, a cada dia, aumentando a necessidade da produção de alimento.

O objetivo deste estudo foi avaliar o risco de salinidade e de sodicidade no solo pelo manejo da irrigação, por meio do incremento nos valores de CEes e RAS de uma área irrigada em relação a uma área de mata nativa, como também o efeito da chuva na lixiviação desses sais ao longo do período estudado, em dois distritos de irrigação da Bacia do Acaraú, Ceará.

\section{MATERIAL E MÉTODOS}

O estudo foi desenvolvido em duas áreas da Bacia do Acaraú (Figura 1), correspondendo aos Distritos Irrigados Baixo Acaraú (DIBAU) e Araras Norte (DIPAN). 


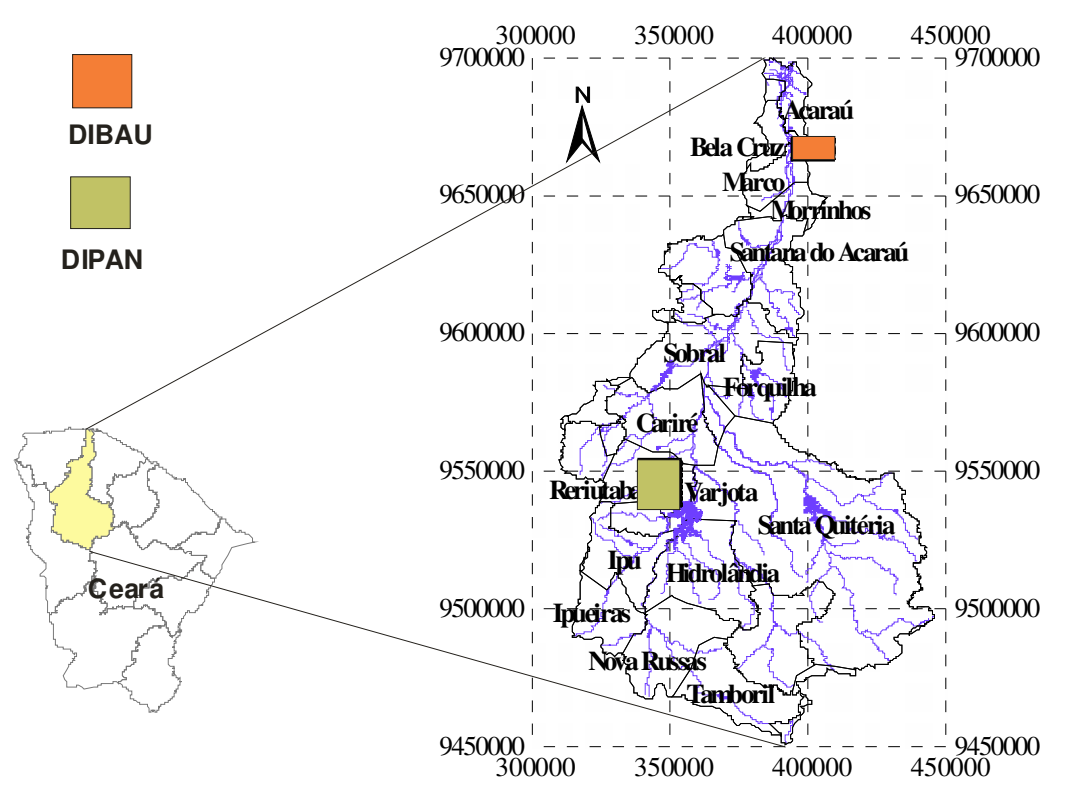

FIGURA 1. Localização do DIBAU e do DIPAN na Bacia do Acaraú, Ceará. Location of DIBAU and DIPAN in Acaraú basin, Ceará, Brazil.

Para o DIBAU, foram coletadas amostras de solo em área explorada com a cultura da bananeira (Musa sp), variedade Prata, irrigada por microaspersão, e que se encontrava, no início do estudo, com 12 meses de exploração. Anualmente, era adicionada $1,0 \mathrm{t} \mathrm{ha}^{-1}$ de calcário dolomítico. Essa área é caracterizada como lote de Pequeno Produtor Qualificado, com área total de 8,0 ha. $\mathrm{O}$ Distrito de Irrigação Baixo Acaraú dista aproximadamente $220 \mathrm{~km}$ de Fortaleza e está situado entre as coordenadas $3^{\circ} 1^{\prime}$ e $3^{\circ} 22^{\prime}$ sul e entre os paralelos $40^{\circ} 1^{\prime}$ e $40^{\circ} 22^{\prime}$ oeste. A região apresenta, de acordo com a classificação de Köeppen, clima do tipo Aw' (tropical chuvoso), com chuvas de outono, e temperatura média mensal do mês mais frio sempre superior a $18{ }^{\circ} \mathrm{C}$. Apresenta, ainda, evapotranspiração anual de $1.600 \mathrm{~mm}$, e velocidade média dos ventos, de $3,0 \mathrm{~m} \mathrm{~s}^{-1}$. Em geral, os solos do DIBAU são profundos, bem drenados, de textura leve e muito permeáveis. Esses pertencem, predominantemente, às classes dos Argissolos, Latossolos e Neossolos (EMBRAPA, 1999).

No DIPAN, foram coletadas amostras de solo em área explorada com a cultura da videira (Vitis vinifera L.), variedade Benitaka, irrigada por microaspersão, e que se encontrava, no início deste estudo, com dois anos de exploração. Anualmente, eram adicionadas $2,5 \mathrm{t} \mathrm{ha}^{-1}$ de calcário dolomítico e 0,24 t ha ${ }^{-1}$ de nitrato de cálcio. Essa área é classificada como lote de categoria Técnico Agrícola, com área total de 11,0 ha. Esse Distrito dista, aproximadamente, $300 \mathrm{~km}$ de Fortaleza e situa-se entre as coordenadas 4\% $4^{\prime}$ e $4^{\circ} 13^{\prime}$ sul e $40^{\circ} 28^{\prime}$ e $40^{\circ} 36^{\prime}$ oeste (Figura 1). A região apresenta, de acordo com a classificação de Köeppen, clima do tipo BSw'h', semi-árido quente, com chuvas de verão-outono, e temperaturas superiores a $18{ }^{\circ} \mathrm{C}$. A evapotranspiração anual é em torno de $1.942 \mathrm{~mm}$, e a velocidade média dos ventos, de $0,58 \mathrm{~m} \mathrm{~s}^{-1}$. A região do DIPAN está localizada sobre uma mancha de solos bruno não-cálcicos (Luvissolos), com parcela mínima sobre o Latossolo Vermelho-Amarelo.

Os totais anuais das precipitações pluviométricas foram obtidos do banco de dados da FUNCEME (2006), compreendendo os seguintes pontos de observação: o posto de coleta da cidade de Marco, representativo do DIBAU, e o posto de coleta da cidade de Varjota, representando a região do DIPAN.

As campanhas de coleta das amostras de solo foram realizadas bimestralmente, no período de março de 2003 a setembro de 2005, nas áreas irrigadas e em suas respectivas matas nativas. As profundidades amostradas corresponderam às camadas de 0-0,30 m;0,30-0,60 m;0,60-0,90 m e 
0,90-1,20 m. Na área irrigada, para cada camada, os solos foram coletados em quatro pontos distribuídos aleatoriamente, dando origem a uma amostra composta representativa de cada camada, enquanto, para a mata nativa, realizou-se apenas uma amostra em cada camada. Os solos foram acondicionados em sacos plásticos, identificados, e em seguida enviados ao Laboratório de Água e Solo da Embrapa Agroindústria Tropical para a determinação dos seguintes parâmetros: $\mathrm{pH}$, CEes, $\mathrm{Ca}^{2+}, \mathrm{Mg}^{2+}, \mathrm{Na}^{+}, \mathrm{K}^{+}$e $\mathrm{Cl}^{-}$. A metodologia utilizada foi a recomendada por RICHARDS (1954). A água utilizada na irrigação, em ambos os distritos, é classificada com $\mathrm{C}_{1} \mathrm{~S}_{2}$ (Tabela 1), o que caracteriza água de baixa salinidade e concentração média de sódio (HOLANDA \& AMORIM, 1997). A água que abastece o DIBAU e o DIPAN provém, respectivamente, da Barragem Santa Rosa e do Açude Público Paulo Sarasate.

TABELA 1. Valores médios da análise das águas no período de estudo, com respectiva classificação. Average values of studied parameters and water classification.

\begin{tabular}{|c|c|c|c|c|c|c|c|c|c|c|}
\hline \multirow{2}{*}{ Fonte Hídrica } & $\mathrm{CE}$ & RAS $^{0}$ & $\mathrm{Na}^{+}$ & $\mathrm{K}^{+}$ & $\mathrm{Ca}^{0}$ & $\mathrm{Mg}^{2+}$ & $\mathrm{Cl}^{-}$ & $\mathrm{HCO}_{3}^{-}$ & $\mathrm{SO}_{4}^{-}$ & \multirow{2}{*}{ Classe } \\
\hline & $\mathrm{dS} \mathrm{m}^{-1}$ & - & & -- & - & $\mathrm{mmol}_{\mathrm{c}}$ & & & --- & \\
\hline & 0,20 & 1,14 & 1,06 & 0,18 & 1,27 & 0,47 & 0,77 & 1,35 & 0,08 & $\mathrm{C}_{1} \mathrm{~S}_{2}$ \\
\hline Barragem Santa Rosa & 0,29 & 1,54 & 1,51 & 0,17 & 1,30 & 0,62 & 1,41 & 1,46 & 0,14 & $\mathrm{C} 1 \mathrm{~S}_{2}$ \\
\hline
\end{tabular}

$\mathrm{RAS}^{0}$ (RAS corrigida) e $\mathrm{Ca}^{0}$ (Cálcio corrigido) de acordo com University of Califórnia Committee of Consultants UCCC.

A inspeção do impacto da irrigação nos dois distritos de irrigação considerados nesta pesquisa fundamentou-se em análise comparativa da concentração dos sais totais, representados pela CEes, e do risco de sodicidade indicado pela RAS, do extrato de saturação. Para identificar se os sais adicionados ao solo pelo manejo da irrigação geravam impacto estatisticamente significativo, aplicou-se o teste de Wilcoxon.

\section{RESULTADOS E DISCUSSÃO}

De modo geral, houve incremento dos valores de CEes da área irrigada em relação à mata nativa (Figura 2B), nos solos do DIBAU. Foi observado também que, no período chuvoso do ano de 2004, houve intensa redução nesse incremento, no qual os valores da área irrigada se equivaleram aos da mata nativa, diferentemente dos valores observados nos outros períodos estudados. Isso se explica pela lixiviação dos sais adicionados ao solo da área irrigada, em decorrência do total de chuva precipitada nesse ano, que atingiu $1.053,50 \mathrm{~mm}$, sendo $17 \%$ superior à média anual da região, expressando a ação da chuva na lavagem dos solos. Além disso, 56,6\% (596,00 mm) dessa pluviosidade ocorreu em apenas um mês, janeiro de 2004 (Figura 2A). Esses resultados estão de acordo com VAN HOORN (1971), que afirma que a lixiviação no perfil do solo é maior quando uma dada altura pluviométrica, que ocorreria em um longo período de tempo, concentra-se em um curto espaço de tempo.

Para o ano de 2005, de maneira geral, verificou-se que os incrementos são bem superiores aos observados durante o ano de 2004. Nesse mesmo período, os percentuais de incremento equivalemse aos valores observados em 2003. Tal fato pode ser atribuído à adição de sais, tanto pela água de irrigação, como pela utilização de adubos químicos como fonte de nutrientes para as plantas. Outro fator determinante para a elevação desse incremento, em 2005, é que, diferentemente do período chuvoso de 2004, o total precipitado em $2005(514,50 \mathrm{~mm})$ foi 42\% inferior à média anual da região, não sendo suficiente para promover a lixiviação de sais na mesma intensidade do ano anterior (2004).

Os maiores incrementos (em torno de 300\%) ocorreram na camada de 0,60-0,90 m, em julho de 2003. Essa maior concentração nas camadas mais profundas confirma a ação das chuvas na lixiviação dos sais adicionados ao solo pelo manejo da irrigação no DIBAU. Em 2005, de modo geral, os maiores incrementos ocorreram na camada de 0,30-0,60 m, em decorrência das menores alturas pluviométricas registradas nesse ano, como citado anteriormente. Esses resultados 
confirmam os encontrados por PEREIRA et al. (1986) e BEN-HUR et al. (2001), os quais mostram a ação da chuva na lixiviação dos sais. Os incrementos de sais totais registrados na área irrigada do DIBAU foram altamente significativos pelo teste de Wilcoxon, para as camadas de $0-0,30 \mathrm{~m} ; 0,30$ 0,60 m e 0,60-0,90 m (Tabela 2). Nessas camadas, apesar de os incrementos se mostrarem altamente significativos, os valores de CEes (Tabela 3) apresentaram-se bem inferiores ao limite de classificação de solos salinos (CEes $>4,0 \mathrm{dS} \mathrm{m}^{-1}$ ).

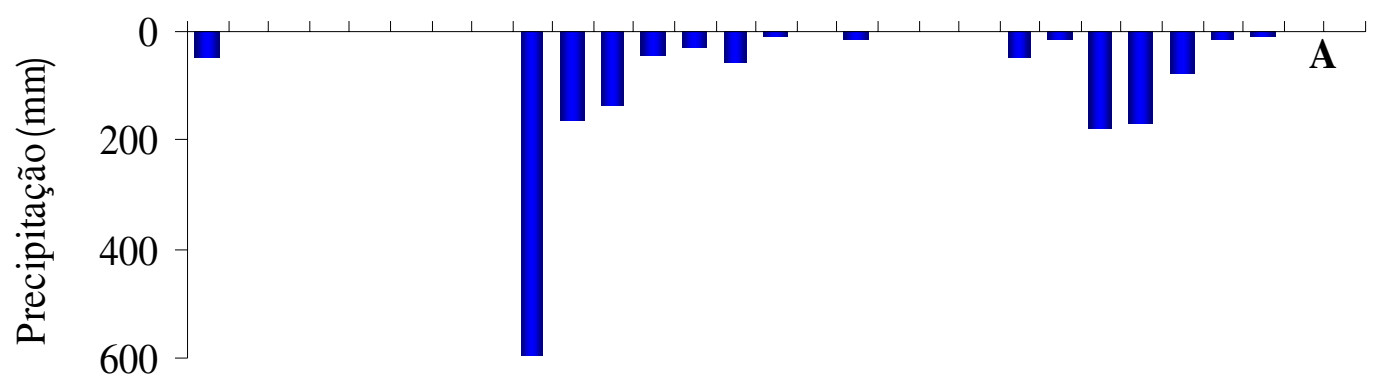

B

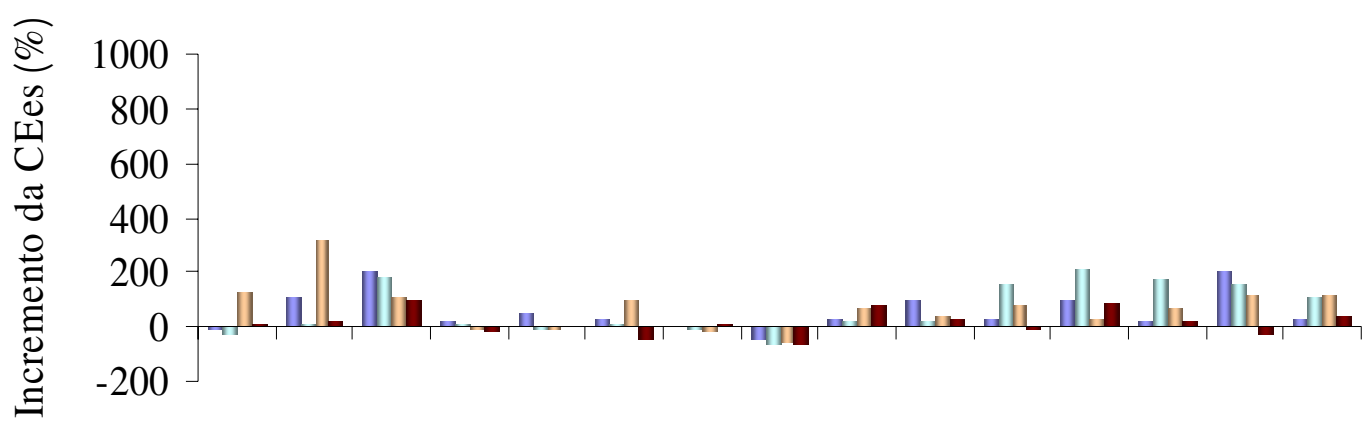

C

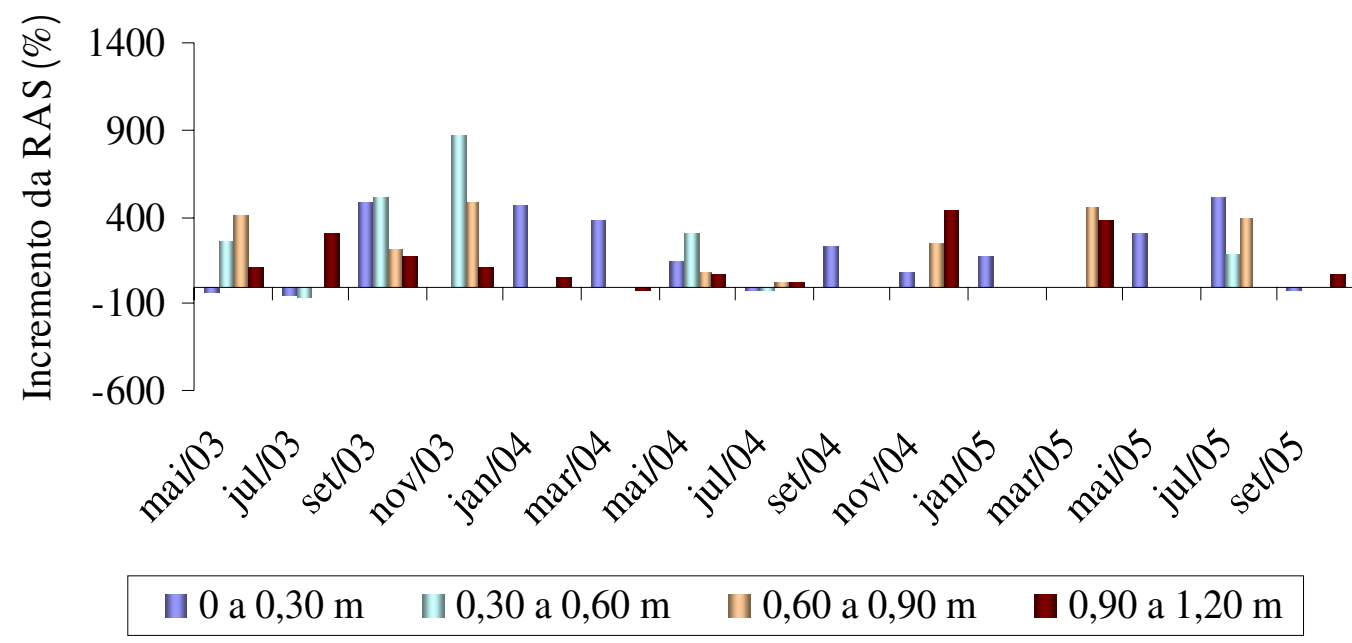

FIGURA 2. Ação da chuva na lixiviação dos sais adicionados pelo manejo da irrigação nas áreas irrigadas do DIBAU, Ceará. Rainfall lixiviation of added salts by irrigation management in DIBAU áreas, Ceará, Brazil. 
TABELA 2. Teste de Wilcoxon para os incrementos de CEes, nas camadas e períodos estudados, no DIBAU, Ceará. Wilcoxon paired test of EC increments in the studied soil layers in DIBAU, Ceará, Brazil.

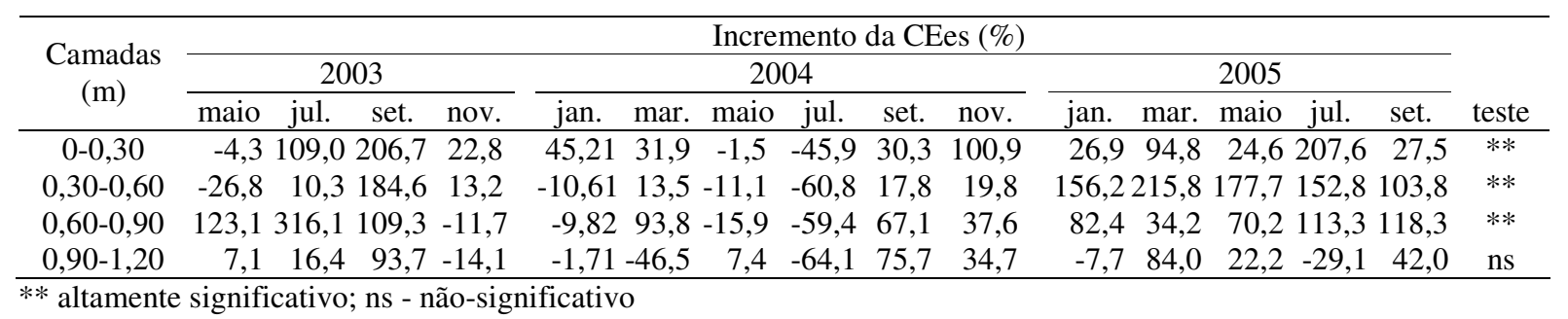

TABELA 3. Valores médios da CEes, RAS, $\mathrm{Ca}^{2+}, \mathrm{Mg}^{2+}$ e $\mathrm{Na}^{+}$, nas quatro camadas do solo, em área irrigada (AI) e mata nativa (MN), no DIBAU, Ceará. Average values of EC, SAR, $\mathrm{Ca}^{2+}, \mathrm{Mg}^{2+} \mathrm{e} \mathrm{Na}^{+}$of the four soil layer in the irrigated area (AI) and native vegetation (MN) in DIBAU, Ceará, Brazil.

\begin{tabular}{lcccccccc}
\hline & \multicolumn{2}{c}{$0-0,30 \mathrm{~m}$} & \multicolumn{2}{c}{$0,30-0,60 \mathrm{~m}$} & \multicolumn{2}{c}{$0,60-0,90 \mathrm{~m}$} & \multicolumn{2}{c}{$0,90-1,20 \mathrm{~m}$} \\
& $\mathrm{AI}$ & $\mathrm{MN}$ & AI & MN & AI & MN & AI & MN \\
\hline CEes $\left(\mathrm{dS} \mathrm{m}^{-1}\right)$ & 0,22 & 0,15 & 0,19 & 0,14 & 0,17 & 0,12 & 0,14 & 0,14 \\
$\mathrm{RAS}$ & 3,63 & 1,66 & 3,04 & 1,51 & 4,02 & 1,45 & 3,73 & 1,78 \\
$\mathrm{Ca}^{2+}\left(\mathrm{mmol}_{\mathrm{c}} \mathrm{L}^{-1}\right)$ & 0,12 & 0,12 & $<0,05$ & $<0,05$ & $<0,05$ & $<0,05$ & $<0,05$ & $<0,05$ \\
$\mathrm{Mg}^{2+}\left(\mathrm{mmol}_{\mathrm{c}} \mathrm{L}^{-1}\right)$ & 0,20 & 0,20 & $<0,05$ & 0,11 & $<0,05$ & 0,10 & $<0,05$ & 0,14 \\
$\mathrm{Na}^{+}\left(\mathrm{mmol}_{\mathrm{c}} \mathrm{L}^{-1}\right)$ & 0,99 & 0,49 & 0,61 & 0,38 & 0,54 & 0,34 & 0,61 & 0,52 \\
\hline
\end{tabular}

Para o risco de sodicidade (Figura 2C), verificaram-se incrementos superiores aos registrados em relação ao risco de salinidade (Figura 2B). Os menores incrementos correspondem ao ano de 2004, em decorrência da altura pluviométrica ocorrida naquele ano, como já apresentado e discutido anteriormente. No entanto, o total precipitado não foi suficiente para levar a área irrigada à condição de mata nativa. Os maiores incrementos foram observados na camada de 0,30-0,60 m, no mês de novembro de 2003, alcançando valores em torno de $860 \%$. A RAS (Tabela 3) apresentou valores bem inferiores ao limite de classificação de solos sódico (RAS > 13,0).

Em muitas amostras de solo coletado na área do DIBAU, não foi detectada a presença $\mathrm{Ca}^{2+}$ e/ou $\mathrm{Mg}^{2+}$ como decorrência natural dos solos predominantes dessa região (solos arenosos, com baixa concentração de argila). Como a RAS expressa a concentração de sódio em relação ao cálcio e ao magnésio, essa limitação gerou poucos dados de incremento de RAS, impossibilitando a aplicação do teste de Wilcoxon.

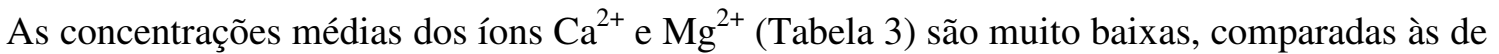
$\mathrm{Na}^{+}$; e baixos valores de cálcio e magnésio aumentam o risco de sodicidade. Nessas condições, pequeno aumento dos valores do íon $\mathrm{Na}^{+}$na área irrigada, em comparação com a mata nativa, resulta em elevado incremento na RAS (Figura 2C), aumentando o risco de sodicidade. Em todas as camadas, as concentrações de cálcio foram muito pequenas, expressando a necessidade de correção do solo. Esses valores são bem inferiores ao limite mínimo para a solução do solo apresentado por AYERS \& WESTCOT (1999); esses comentam que, se a concentração do $\mathrm{Ca}^{2+}$ na solução do solo for inferior a $2 \mathrm{mmol}_{\mathrm{c}} \mathrm{L}^{-1}$, existe alta probabilidade de redução na produtividade das culturas, como também pode manifestar deficiência de cálcio na planta. Observou-se, também, que a concentração de $\mathrm{Mg}^{2+}$ foi superior à do cálcio, o que promove maior predisposição do efeito do íon sódio, ou seja, determinado valor de RAS é ligeiramente mais perigoso quando a proporção $\mathrm{Ca}^{2+} / \mathrm{Mg}^{2+}<1$.

Em geral, houve incremento dos valores de CEes durante o período estudado nos solos do DIPAN (Figura 3B). Para o período chuvoso de 2004, não se identificou redução de incremento, comparado a 2003, apesar de, nesse período, a altura pluviométrica anual ter sido de $1.128,00 \mathrm{~mm}$, 
$41 \%$ a mais do que os valores médios observados para a região do DIPAN. Esses resultados equivalem aos observados por ANDRADE et al. (2004), os quais constataram que precipitação 62\% superior à média anual não foi suficiente para promover lixiviação significativa dos sais adicionados pela irrigação, em solos da Chapada do Apodi, Ceará. Atribuiu-se tal fato ao tipo de solo predominante nesse distrito de irrigação, o qual não apresenta boa drenagem.

Em 2005, registraram-se elevados incrementos, comparados aos anos de 2003 e 2004, ocorrendo tendência de aumento contínuo. O último mês do período estudado foi o que mostrou valores mais críticos. Tal fato está relacionado aos sais adicionados, tanto pela água de irrigação, quanto pelos fertilizantes minerais, em torno de 4,6 $\mathrm{t} \mathrm{ha}^{-1}$ ano $^{-1}$, utilizados na realização das adubações. A influência do inadequado manejo de fertilizantes minerais em áreas irrigadas foi registrada também por CAUSAPÉ et al. (2004) e FENG et al. (2005). Outra agravante é a reduzida lixiviação de sais devido à menor intensidade pluviométrica registrada em 2005 (Figura 3A), a qual se apresentou $20 \%$ inferior à média da região. Os maiores incrementos ocorreram nos meses que apresentaram ausência total de precipitações pluviométricas.

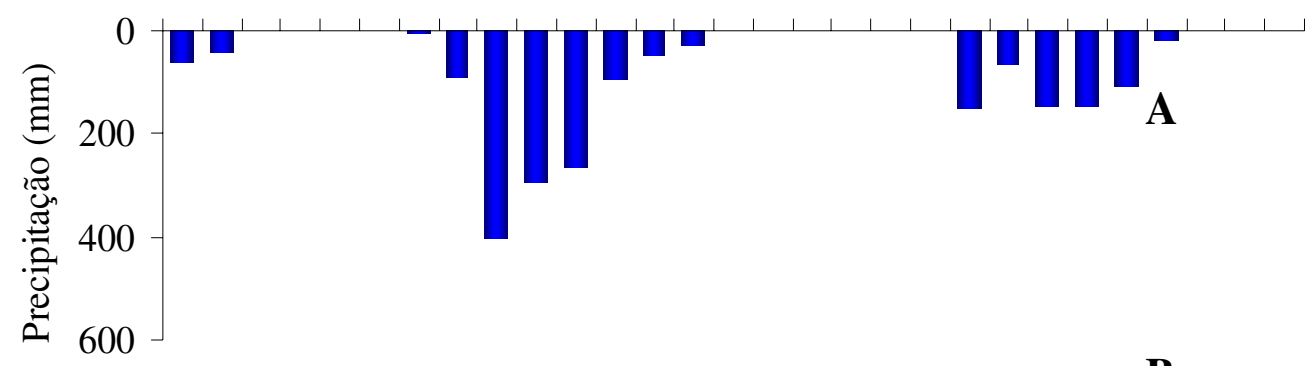

B
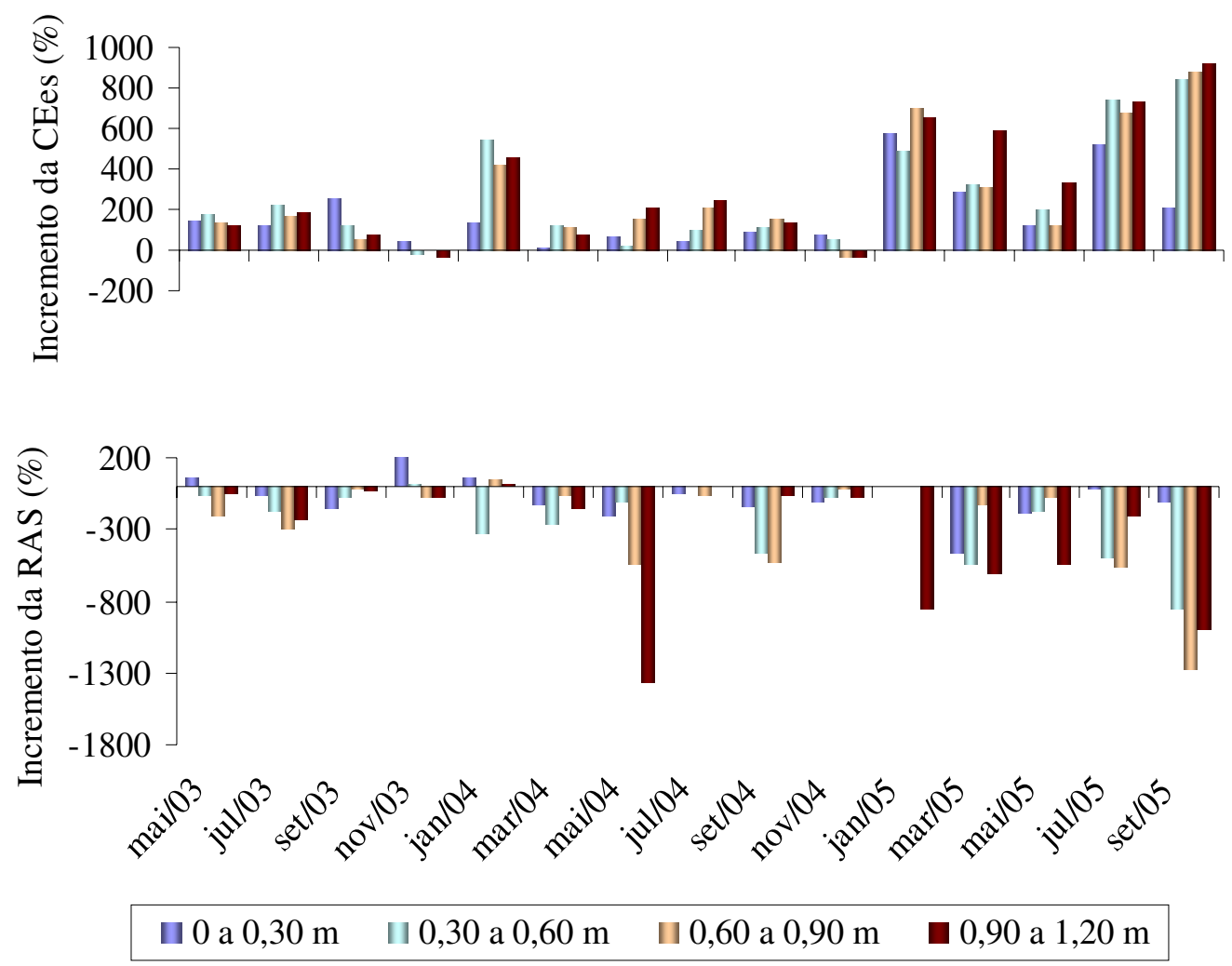

FIGURA 3. Ação da chuva na lixiviação dos sais adicionados pelo manejo da irrigação nas áreas irrigadas do DIPAN, Ceará. Rainfall lixiviation of added salts by irrigation management in DIPAN áreas, Ceará, Brazil. 
De modo geral, os incrementos mostraram-se sempre maiores nas camadas inferiores do solo estudado, principalmente em 2005, quando atingiram valores superiores a $900 \%$ em setembro. Acredita-se que os sais foram deslocados para as citadas camadas por ocasião da aplicação de lâmina excessiva de água, expressando a necessidade de estudo da lâmina de água aplicada no DIPAN. Resultados semelhantes foram encontrados por MEIRELES et al. (2003), que constataram aumento na CEes de 10 vezes em relação à salinidade da mata nativa. As diferenças registradas entre os valores da CEes da área irrigada e os da mata nativa (Tabela 4), para todas as camadas estudadas, foram altamente significativas pelo teste de Wilcoxon, comprovando a adição de sais ao solo. Apesar de os incrementos mostrarem-se altamente significativos, os valores de CEes (Tabela 5) não classificam os solos do DIPAN como salinos (CEes $\left.>4,0 \mathrm{dS} \mathrm{m}^{-1}\right)$.

TABELA 4. Teste de Wilcoxon para os incrementos de CEes e RAS, nas camadas e nos períodos estudados, no DIPAN, Ceará. Wilcoxon paired test of EC and SAR increments in the studied soil layers in DIPAN, Ceará, Brazil.

\begin{tabular}{|c|c|c|c|c|c|c|c|c|c|c|c|c|c|c|c|c|}
\hline \multirow{3}{*}{$\begin{array}{c}\text { Camadas } \\
(\mathrm{m})\end{array}$} & \multicolumn{15}{|c|}{ Incremento de CEes (\%) } & \multirow[b]{3}{*}{ Teste } \\
\hline & \multicolumn{4}{|c|}{2003} & \multicolumn{6}{|c|}{2004} & \multicolumn{5}{|c|}{2005} & \\
\hline & maio & jul. & set. & nov. & jan. & mar. & maio & jul. & set. & nov. & jan. & mar. & maio & jul. & set. & \\
\hline $0-0,30$ & 142,0 & 122,7 & 255,7 & 49,4 & 130,2 & 12,0 & 70,9 & 41,1 & 93,1 & 79,1 & 572,9 & 285,0 & 122,3 & 517,0 & 208,3 & $* *$ \\
\hline $0,30-0,60$ & 179,6 & 219,2 & 117,4 & $-25,0$ & 542,8 & 125,7 & 20,9 & 99,4 & 106,1 & 54,1 & 490,4 & 325,8 & 200,8 & 740,6 & 841,2 & $* *$ \\
\hline $0,60-0,90$ & 133,0 & 168,4 & 53,0 & 2,8 & 421,0 & 110,4 & 150,6 & 208,0 & 154,6 & $-35,3$ & 698,7 & 313,2 & 123,8 & 681,5 & 880,0 & $* *$ \\
\hline $0,90-1,20$ & 123,3 & 183,9 & 83,2 & $-38,5$ & 452,8 & 78,1 & 209,6 & 249,9 & 132,4 & $-35,0$ & 655,8 & 587,8 & 335,6 & 736,7 & 917,0 & $* *$ \\
\hline \multicolumn{17}{|c|}{ Incremento de RAS (\%) } \\
\hline $0-0,30$ & 52,9 & $-66,6$ & $-159,5$ & 194,8 & 55,3 & $-119,0$ & $-196,0$ & $-53,0$ & $-145,7$ & $-111,7$ & 6,1 & $-471,9$ & $-188,6$ & $-24,4$ & $-104,6$ & ns \\
\hline $0,30-0,60$ & $-60,3$ & $-171,7$ & $-82,0$ & 21,6 & $-331,6$ & $-268,8$ & $-108,5$ & 4,1 & $-469,0$ & $-72,1$ & $-550,9$ & $-169,2$ & $-500,5$ & $-849,6$ & - & ns \\
\hline $0,60-0,90$ & $-196,5$ & $-294,0$ & $-11,4$ & $-83,1$ & 48,8 & $-59,9$ & $-537,9$ & $-63,3$ & $-530,4$ & $-10,1$ & - & $-132,7$ & $-71,6$ & $-560,9-$ & 1279,9 & ns \\
\hline $0,90-1,20$ & $-48,8$ & $-241,6$ & $-28,9$ & $-76,7$ & 18,2 & $-150,5-$ & 1362,1 & - & $-58,6$ & $-81,0$ & $-858,2$ & $-602,5$ & $-549,5$ & $-205,7$ & $-989,6$ & ns \\
\hline
\end{tabular}

TABELA 5. Valores médios da CEes, RAS, $\mathrm{Ca}^{2+}, \mathrm{Mg}^{2+}$ e $\mathrm{Na}^{+}$, nas quatro camadas do solo, em área irrigada (AI) e mata nativa (MN), no DIPAN, Ceará. Average values of EC, SAR, $\mathrm{Ca}^{2+}, \mathrm{Mg}^{2+} \mathrm{e} \mathrm{Na}^{+}$of the four soil layer in the irrigated area (AI) and native vegetation (MN) in DIPAN, Ceará, Brazil.

\begin{tabular}{lcccccccc}
\hline & \multicolumn{2}{c}{$0-0,30 \mathrm{~m}$} & \multicolumn{2}{c}{$0,30-0,60 \mathrm{~m}$} & \multicolumn{2}{c}{$0,60-0,90 \mathrm{~m}$} & \multicolumn{2}{c}{$0,90-1,20 \mathrm{~m}$} \\
& AI & MN & AI & MN & AI & MN & AI & MN \\
\hline CEes $\left(\mathrm{dS} \mathrm{m}^{-1}\right)$ & 0,84 & 0,31 & 0,68 & 0,23 & 0,62 & 0,22 & 0,61 & 0,21 \\
$\mathrm{RAS}$ & 0,61 & 0,93 & 0,61 & 1,63 & 0,57 & 1,63 & 0,57 & 2,15 \\
$\mathrm{Ca}^{2+}\left(\mathrm{mmol}_{\mathrm{c}} \mathrm{L}^{-1}\right)$ & 2,76 & 0,64 & 1,73 & 0,51 & 1,70 & 0,57 & 1,89 & 0,43 \\
$\mathrm{Mg}^{2+}\left(\mathrm{mmol}_{\mathrm{c}} \mathrm{L}^{-1}\right)$ & 1,95 & 0,41 & 1,09 & 0,29 & 1,01 & 0,22 & 1,01 & 0,18 \\
$\mathrm{Na}^{+}\left(\mathrm{mmol}_{\mathrm{c}} \mathrm{L}^{-1}\right)$ & 0,71 & 0,54 & 0,58 & 0,62 & 0,57 & 0,59 & 0,63 & 0,67 \\
\hline
\end{tabular}

Para o risco de sodicidade dos solos do DIPAN, observa-se que, de modo geral, não ocorreram incrementos, registrando, em quase sua totalidade, menor valor da RAS na área irrigada (Figura 3C), não havendo diferença significativa entre os valores das duas áreas estudadas (Tabela 3). Esse menor risco de sodicidade pode ser explicado pelas concentrações de $\mathrm{Ca}^{2+} \mathrm{e} \mathrm{Mg}^{2+}$, registradas na área irrigada, serem sempre superiores aos valores observados na mata nativa (Tabela 5) e, tanto nessa quanto na área irrigada, os valores não classificam o solo como sódico (RAS > 13,0). Tal fato deveu-se à contínua aplicação de calcário no solo, como também às fontes alternativas de cálcio e magnésio para suprirem irregularidades nutricionais da cultura da uva.

\section{CONCLUSÕES}

No DIBAU, a precipitação levou a área irrigada à condição de mata nativa, para o parâmetro CEes, mas não com relação à RAS. 
Os maiores incrementos de CEes, para o DIPAN, foram observados nas camadas inferiores, expressando a lixiviação dos sais.

Os solos do DIPAN apresentaram maior risco de salinidade em todas as camadas estudadas.

No DIBAU (solos arenosos), o processo de lixiviação foi determinado pelo total precipitado, enquanto, no DIPAN (solos pesados), o processo foi definido pelo solo.

\section{REFERÊNCIAS}

ANDRADE, E.M.; D'ALMEIDA, D.M.B.A.; MIERELES, A.C.M.; LEMOS FILHO, L.C.A.; DE ARRUDA, F.E.R. Evolução da concentração iônica da solução do solo em áreas irrigadas na Chapada do Apodi, Ceará. Revista Ciência Agronômica, Fortaleza, v.35, n.1, p.9-16, jan./jun. 2004.

AYERS, R.S.; WESTCOT, D.W. A qualidade da água na agricultura. Tradução de GHEYI, H.R.; MEDEIROS, J.F.; DAMASCENO, F.A.V. Campina Grande: UFPB, 1999. 153 p. (Estudos FAO: Irrigação e Drenagem, 29)

BEN-HUR, M.; LI, F.H.; KEREN, R.; RAVINA, I.; SHALIT, G. Water and salt distribution in a field irrigated with marginal water under high water table conditions. Soil Science American Journal, Madison, v.65, n.1, p.191-8, jan. 2001.

BERNARDO, S. Impacto ambiental da irrigação no Brasil. In: SILVA, D.D. da; PRUSKI, F.F. (Ed.) Recursos hídricos e desenvolvimento sustentável da agricultura. Viçosa: MMA/SRH/ ABEAS/UFV, 1997. 252 p.

CAUSAPÉ, J.; QUÍLEZ, D.; ARAGUÉS, R. Assessment of irrigation and environmental quality at the hydrological basin level II. Salt and nitrate loads in irrigation return flows. Agricultural Water Management, Amsterdam, v.70, n.3, p.211-18, dez. 2004.

D’ALMEIDA, D.M.B.A. Risco de salinização de um Cambissolo na Chapada do Apodí - CE. 2002. 68 f. Dissertação (Mestrado em Irrigação e Drenagem) - Universidade Federal do Ceará, Fortaleza, 2002.

D’ALMEIDA, D.M.B.A.; ANDRADE, E.M.; MEIRELES, A.C.M. Importância relativa dos íons na salinidade de um Cambissolo na Chapada do Apodi, Ceará. Engenharia Agrícola, Jaboticabal, v.25, n.3, p.615-21, 2005.

EMBRAPA. EMPRESA BRASILEIRA DE PESQUISA AGROPECUÁRIA. Centro Nacional de Pesquisa de Solos. Sistema brasileiro de classificação de solos. Rio de Janeiro, 1999. 412 p.

FENG, Z.Z.; WANG, X.K.; FENG, Z.W. Soil N and salinity leaching after the autumn irrigation and its impacto $\mathrm{n}$ groundwater in Hetao Irrigation District, China. Agricultural Water Management, Amsterdam, v.71, n.1, p.131-43, 2005.

FUNCEME. FUNDAÇÃO CEARENSE DE METEOROLOGIA E RECURSOS HÍDRICOS. Monitoramento hidroambiental das chuvas. Disponível em: http://www.funceme.br/DIPAN/index.htm. Acesso em: 6 fev. 2006.

HOLANDA, J.S.; AMORIM, J.R.A. Qualidade da água para irrigação In: GHEYI, H.R.; QUEIROZ, J.E.; MEDEIROS, J.F. de (Ed.). Manejo e controle da salinidade na agricultura irrigada. Campina Grande: UFPB, 1997. cap. 5, p.137-69.

LIMA, L.A. Efeitos de sais no solo e na planta. In: GHEYI, H.R.; QUEIROZ, J.E.; MEDEIROS, J.F. de (Ed.). Manejo e controle da salinidade na agricultura irrigada. Campina Grande: UFPB, 1997. p.113-33.

MEIRELES, A.C.M.; ANDRADE, E.M.; CRUZ, M.G.M.; LEMOS FILHO, L.C.A. Avaliação do impacto da fertirrigação em Cambissolos na Chapada do Apodi, Ceará. Revista Ciência Agronômica, Fortaleza, v.34, n.2, p.207-12, ago./dez. 2003. 
ONGLEY, E.D. Controle da poluição da água pelas atividades agrícolas. Tradução de GHEYI, H.R.; DAMASCENO, F.A.V.; BRITO, L. T. de L. Campina Grande: UFPB, 2000.92 p. (Estudos FAO: Irrigação e Drenagem, 55).

PEREIRA, O.J.; MATIAS FILHO, J.; ANDRADE, E.M. Variação do teor de sais no solo irrigado por aspersão e ação da chuva na sua lixiviação. Revista Ciência Agronômica, Fortaleza, v.17, n.1, p.61-5, jun. 1986.

PIZARRO, F. Drenage agrícola y recuperacion de suelos salinos. Madrid: Editorial Agrícola Española, 1978. 525 p.

RICHARDS, L.A. Diagnosis and improvement of saline and alkali soils. Washington: US Departament of Agriculture, 1954. 160 p. (USDA Agricultural Handbook, 60).

SILVA FILHO, S.B.; CAVALCANTE, L.F.; OLIVEIRA, F.A.; LIMA, E.M.; COSTA, J.R.M.

Monitoramento da qualidade da água e acúmulo de sais no solo pela irrigação. Irriga, Botucatu, v.5, n.2, p.112-15, maio/ago. 2000.

SMEDEMA, L.K.; SHIATI, K. Irrigation and salinity: a perspective review of the salinity hazards of irrigation development in the arid zone. Irrigation and Drainage Systems, Amsterdam, v.16, n.2, p.161-74, maio. 2002.

SOUZA, L.C. de; QUEIROZ, J.E.; MEDEIROS, J.F.; GHEYI, H.R. Variabilidade espacial da salinidade de um solo aluvial no semi-árido paraibano. Revista Brasileira de Engenharia Agrícola e Ambiental, Campina Grande, v.4, n.2, p.35-40, jan./abr. 2000.

VAN HOORN, J.W. Quality of irrigation water, limits of use of long-term effects. In: SALINITY SEMINAR, BAGHIDAD. Rome: FAO, 1971. p.117-135. (Irrigation and Drainage Paper, 7). 